\title{
A NOTE ON NEGATIVE CUSTOMERS, GI/G/1 WORKLOAD, AND RISK PROCESSES
}

\author{
RICHARD J. BOUCHERIE* \\ Department of Econometrics, University of Amsterdam \\ Roetersstraat 11, 1018 WB Amsterdam, The Netherlands \\ ONNO J. BOXMA \\ CWI, P.O. Box 94079 \\ 1090 GB Amsterdam, The Netherlands \\ and \\ Tilburg University, Faculty of Economics \\ P.O. Box 90153 \\ 5000 LE Tilburg, The Netherlands \\ KARL SIGMAN \\ Department of Industrial Engineering and Operations Research \\ Columbia University \\ 500 West 120th Street \\ New York, New York 10027-6699
}

\begin{abstract}
This note illustrates that a combination of the approach in our previous papers (Boucherie and Boxma, 1996, Probability in the Engineering and Informational Sciences 10: 261-277; Jain and Sigman, 1996, Probability in the Engineering and Informational Sciences 10: 519-531) directly leads to a Pollaczek-Khintchine form for the workload in a queue with negative customers. The same technique is also applied to risk processes with lump additions.
\end{abstract}

\section{INTRODUCTION}

The workload in queues with work removal via negative customers has been analyzed by Boucherie and Boxma [2] and Jain and Sigman [8]. Previous

* Research made possible by a fellowship of the Royal Netherlands Academy of Arts and Sciences. 
research on negative customers had dealt with customer removal as opposed to work removal (see, e.g., Gelenbe $[6,7])$; allowing work removal is particularly useful when the content being modeled is a fluid (such as in dams) or approximately so (such as in information systems). The special feature of this model is the occurrence of downward jumps in workload due to a negative arrival (removing a random amount of work). This is in addition to the upward jumps caused by the service times of regular (positive) customers. A similar phenomenon can be observed in risk processes. In a standard risk process, a risk premium is earned at a constant rate, and claims result in downward jumps in the reserve. In the life annuity business, this situation is reversed. Here a net premium is paid to the policyholders, and the death of a policyholder results in an upward jump in the reserve: the reserve is placed at the disposal of the company. A general risk process incorporating both types of insurance therefore has both positive and negative jumps (Cramér [4]).

One of the goals of the present note is to demonstrate how a simple transformation reduces a risk process with both upward and downward jumps to a standard risk process with only downward jumps. This is done in Section 3. This transformation idea was introduced by Boucherie and Boxma [2], where it was used to transform the workload process in the $\mathrm{M} / \mathrm{G} / 1$ queue with additional negative customers into the workload process of a standard GI/G/1 queue without negative customers. In Section 2 of the present note, we further explore the possibilities opened by this transformation. We show how it yields an immediate explanation of the remarkable occurrence, first observed by Jain and Sigman $[8,9]$, of a generalized Pollaczek-Khintchine form for the Laplace-Stieltjes transform of the workload distribution in the $\mathrm{M} / \mathrm{G} / 1$ queue with negative customers.

\section{A TRANSFORMATION}

Consider the M/G/1 queue with additional removal of work as studied by Boucherie and Boxma [2] and Jain and Sigman [8]. Customers arrive according to a Poisson process with rate $\lambda^{+}$. Their service requirements $\left\{B_{n}\right\}$ are independent and identically distributed (i.i.d.) with distribution $B(\cdot)$, finite mean $\beta$, and Laplace-Stieltjes transform (LST) $\beta(s)$. We shall refer to these customers as ordinary or positive customers. In addition to the ordinary customers, and independent of them, negative customers arrive at the queue according to a Poisson process with rate $\lambda^{-}$. These negative customers reduce the amount of work in the queue according to a distribution $C(\cdot)$, with mean $\gamma$ and LST $\gamma(s)$. These reduction amounts are denoted by $\left\{C_{n}\right\}$ and are assumed i.i.d. Assume that $\lambda^{+} \beta<1+\lambda^{-} \gamma$; this is the stability condition for this $M / G / 1$ generalization $[2,8]$. One can even allow the case in which negative customers always remove all the work present; this is the so-called disaster model $[2,9]$.

Let $V(t)$ denote the workload at time $t, V$ the steady-state workload, and $V_{a}$ the steady-state workload as found by arriving ordinary customers. Let $t_{n}$ denote the arrival epoch of the $n$th positive customer, with $t_{0} \stackrel{\text { def }}{=} 0$. Let 
$\tau_{n} \stackrel{\text { def }}{=} t_{n+1}-t_{n}$. Then, $\left\{\tau_{n}\right\}$ and $\left\{B_{n}\right\}$ are independent sequences of random variables, and each of these sequences consists of i.i.d. random variables. The amount of work found by the arriving positive customers then satisfies

$$
V\left(t_{n+1}\right)=\max \left(V\left(t_{n}\right)+B_{n}-\tau_{n}-d_{n}, 0\right),
$$

where $d_{n}$ is the amount of work destroyed by the negative customers that arrive during $\tau_{n}$. Let $K_{n}$ denote the number of negative customers arriving during the interarrival time $\tau_{n}$. The amount of work destroyed by the $j$ th negative customer arriving during this interarrival time is denoted by $C_{j}^{n}$ and has distribution $C(\cdot)$. Thus, $\left\{d_{n}\right\}$ is an i.i.d. sequence, where $d_{n}$ has the same distribution as $\sum_{j=1}^{K_{n}} C_{j}^{n}$. It is obvious that $\left\{d_{n}\right\}$ is independent of $\left\{B_{n}\right\}$ but that $\tau_{n}$ and $d_{n}$ are dependent.

The form of Eq. (1) suggests the following transformation, relating the workload at arrival epochs of positive customers to this workload at arrival epochs in a standard GI/G/1 queue [2] (cf. Fig. 1). The amount of work found in the queue by an arriving positive customer in the $M / G / 1$ queue with negative customers corresponds to the waiting time of a customer arriving in a

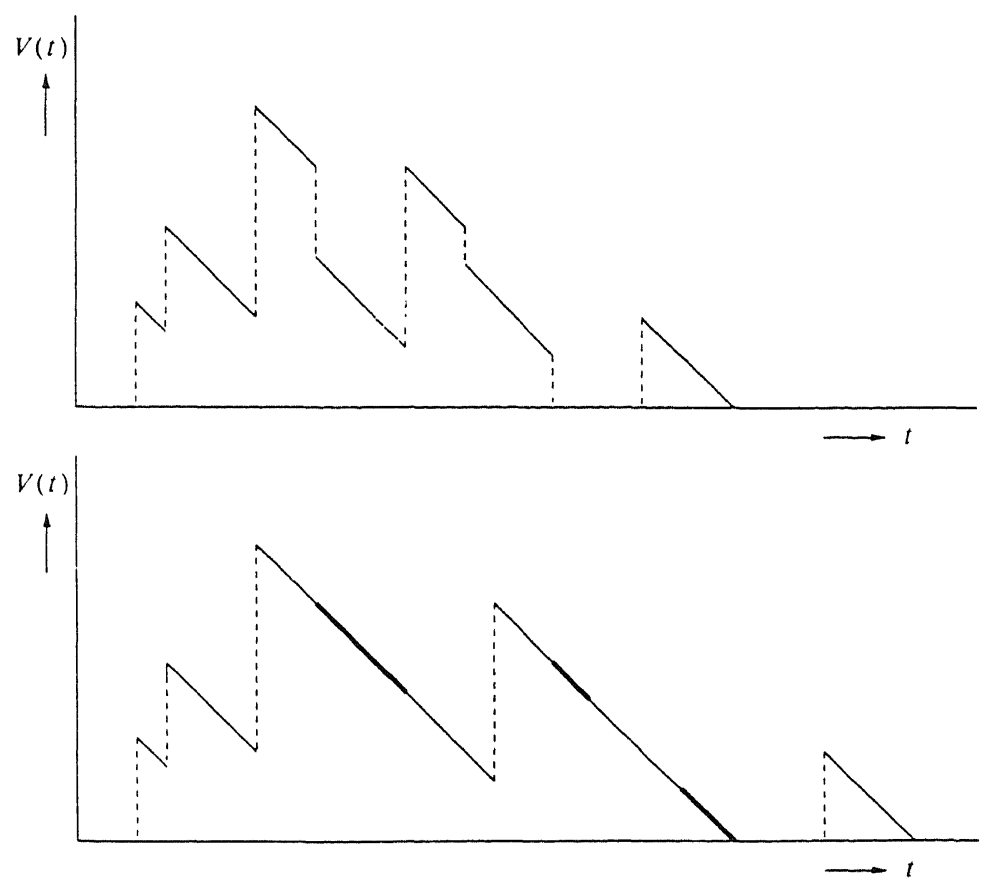

Figure 1. Workload in the $M / G / 1$ queue with negative customers (above) and in the transformed model (below). 
GI/G/1 queue with required service times $\left\{B_{n}\right\}$ and interarrival times $\left\{\tau_{n}^{*}\right\}$, where

$$
\tau_{n}^{*}:=\tau_{n}+d_{n}=\tau_{n}+\sum_{j=1}^{K_{n}^{\prime}} C_{j}^{n} .
$$

Clearly, Eq. (1) is the recurrence relation for the waiting time in a GI/G/1 queue with service times $B_{n}$ but extended interarrival times $\tau_{n}^{*}$. Thus, we conclude that $V_{a}$, and by PASTA also the steady-state workload $V$, has the same distribution as the steady-state waiting time in this $\mathrm{GI} / \mathrm{G} / 1$ queue.

It is widely known that the LST of the latter distribution in the $M / G / 1$ queue has the Pollaczek-Khintchine form

$$
\phi(s)=\frac{1-\nu}{1-\nu \eta(s)}, \quad \operatorname{Re} s \geq 0,
$$

with $\nu$ the traffic load and $\eta(s)=(1-\beta(s)) / \beta s$. It is also known (but perhaps not as widely) that a similar form, with different $\nu$ and $\eta(s)$, even holds for the waiting time LST in the $\mathrm{Gl} / \mathrm{G} / 1$ queue. This follows from random walk theory. Consider the random walk

$$
R_{n}=B_{1}-\tau_{1}^{*}+\cdots+B_{n}-\tau_{n}^{*}, \quad n \geq 1, \quad R_{0}=0,
$$

with i.i.d. increments $B_{i}-\tau_{i}^{*}$. The steady-state waiting time distribution in the first in-first out GI/G/1 queue is that of the maximum $M=\max \left\{R_{n}: n \geq 0\right\}$. By considering the ladder heights, observing that the ladder height process restarts from scratch upon a strictly ascending ladder moment, it follows that the LST of $M$ is of the generalized Pollaczek-Khintchine form of Eq. (3), with $\nu=P\left(R_{n}>0\right.$, for some $\left.n \geq 0\right)$ being the probability of at least one strictly ascending ladder height, and $\eta(s)$ the LST of such a ladder height distribution (conditional on it occurring) (see, e.g., Wolff [15, ch. 9]).

In [8], formula (3) is derived for the model with negative customers by utilizing the preemptive last in-first out discipline (PL) and extending a result of Fakinos [5] (see also Niu [11]). Under PL, $\eta(s)$ represents the LST of the distribution of the remaining service time of the customer found in service by an arrival, say $B^{*}(\cdot)$, and

$$
P\left(Q=n, V_{j} \leq x_{j}, j=1, \ldots, n\right)=(1-\nu) \nu^{n} \prod_{j=1}^{n} B^{*}\left(x_{j}\right),
$$

where $Q$ is the number of customers found by an arrival and $V_{j}$ the remaining service requirement of customer $j, j=1, \ldots, n$. In this context, $\nu=P(V>0)$.

Remark 2.1: The transformation idea of [2], lengthening of interarrival times to take work removal into account, gives direct access to the literature on the Gl/G/1 queue; see, for example, Cohen [3], in particular for the useful detailed results for the case that either the interarrival time distribution or the service time distribution has a rational LST. 
The transformation holds more generally in a $\mathrm{G} / \mathrm{G} / 1$ queue with a stationary sequence $\left\{\left(B_{n}, \tau_{n}\right)\right\}$ of service and interarrival times for positive customers. The transformed sequence becomes the new stationary sequence $\left\{\left(B_{n}, \tau_{n}^{*}\right)\right\}$ as defined by (2) (the new sequence remains stationary because the negative arrivals are Poisson and independent of all else). In the GI/G/1 case where the interarrival times are i.i.d., the LST $\tau(s)$ of interarrival time is transformed into $\tau\left(s+\lambda^{-}(1-\gamma(s))\right)$.

The transformation idea even applies to a $\mathrm{GI} / \mathrm{G} / 1$ queue in which each arriving customer is with probability $p$ a positive customer who requires some service and with probability $1-p$ a negative customer who removes some work; now, $\tau(s)$ transforms into $p \tau(s) /(1-(1-p) \tau(s))$. Of course, in these cases, PASTA may not hold and the distributions of $V$ and $V_{a}$ are typically different.

Remark 2.2: It is known (cf. Cohen [3, p. 282]) that the waiting time distribution in the $\mathrm{GI} / \mathrm{G} / 1$ queue is infinitely divisible. Interestingly, this immediately follows from the form of Eq. (3) and Theorem 12.2.3 in Lukacs [10] (which states that such a form implies infinite divisibility).

\section{RISK PROCESSES WITH LUMP ADDITIONS}

The probability of ruin of a risk process with Poisson arrivals of claims and initial reserve $x$ is related to the tail of the workload distribution in an $M / G / 1$ queue, $P(V>x)$ (e.g., Asmussen and Sigman [1] and Prabhu [12]). Following the transformation used for queues with negative customers, it is natural to pursue a similar transformation for the probability of ruin of a risk process with additional positive jumps, thus simplifying the analysis of such risk processes. That will be done in the present section.

Consider an insurance and life annuity business that starts off initially with $x \geq 0$ units of money and earns a rate 1 premium. Claims occur as a Poisson process at rate $\mu^{-}$with interarrival times $\left\{\tau_{n}\right\}$, and claim sizes $\left\{B_{n}\right\}$ are nonnegative and i.i.d. with distribution $B(\cdot)$. Furthermore, independently, annuity policyholders die and lump sums of money are placed at the disposal of the business according to a Poisson process at rate $\mu^{+}$. These lumps $\left\{C_{n}\right\}$ are nonnegative and i.i.d. with distribution $C(\cdot)$. The total reserve at time $t$ is given by the risk process

$$
X_{x}(t) \stackrel{\text { def }}{=} x+t-\sum_{i=1}^{N-(t)} B_{i}+\sum_{i=1}^{N^{+}(t)} C_{i}, \quad t \geq 0,
$$

where $N^{-}$and $N^{+}$are the Poisson counting processes for claims and lumps, respectively; $X_{x}(0)=x$. Of intrinsic interest is to compute the probability of ruin, $P(\tau(x)<\infty)$, where

$$
\tau(x) \stackrel{\text { def }}{=} \inf \left\{t>0: X_{x}(t)<0\right\},
$$


the time until the business runs out of reserve $\left(\tau(x) \stackrel{\text { def }}{=} \infty\right.$ if $X_{x}(t)$ never enters $(-\infty, 0)$ ). When the model has no lump additions, then it is well known (see, e.g., Prabhu [12]) that

$$
P(\tau(x)<\infty)=P(V>x),
$$

where $V$ is steady-state workload for an M/G/1 queue with arrival rate $\mu^{-}$and service times $\left\{B_{n}\right\}$. It is intuitive that Eq. (4) should also hold for our risk model with lump additions, where $V$ is the steady-state workload in the $M / G / 1$ queue with negative customers in which $\lambda^{+}=\mu^{-}$and $\lambda^{-}=\mu^{+}$. We now show this, following the duality theory from [1].

Observing that ruin can only occur right after a claim epoch (denoted by $t_{n}, n \geq 1$, with $t_{0} \stackrel{\text { def }}{=} 0$ ), we conclude that

$$
\tau(x)=\min \left\{t_{n}>0: X_{n}<0\right\},
$$

where $X_{n} \stackrel{\text { def }}{=} X_{x}\left(t_{n}+\right), n \geq 1$, and $X_{0} \stackrel{\text { def }}{=} x$. But $X_{n}$ is a random walk starting at $x$ with increments $\tau_{n-1}^{*}-B_{n}, n \geq 1$ :

$$
X_{n}=x+\tau_{0}^{*}-B_{1}+\cdots+\tau_{n-1}^{*}-B_{n}, \quad n \geq 1, \quad X_{0}=x,
$$

where $\tau_{n}^{*}$ is defined exactly as in (2), which in the current notation is given by

$$
\tau_{n}^{*}=\tau_{n}+\sum_{j=N^{+}\left(t_{n}\right)+1}^{N^{+}\left(t_{n+1}\right)} C_{j},
$$

and represents the cumulative earnings (interest plus lump sums) in the time interval $\left(t_{n}, t_{n+1}\right]$. Thus, from Example 1 in [1] (and the fact that Poisson processes are time reversible), we conclude that $\left\{X_{n}\right\}$ is the dual of the reflected random walk given by

$$
W_{n+1}=\max \left(W_{n}+B_{n}-\tau_{n}^{*}, 0\right)
$$

and that Eq. (4) holds when $V=W$, the steady state for this reflected random walk. Equation (6), however, is the same recursion as in Eq. (1); so from Section $2, W$ can be identified with the stationary workload in the $\mathrm{M} / \mathrm{G} / 1$ queue with negative customers in which $\lambda^{+}=\mu^{-}$and $\lambda^{-}=\mu^{+}$.

Remark 3.1: The lump additions can be replaced by any Levy process $\{A(t)\}$ with nonnegative increments, in which case Eq. (5) is generalized to $\tau_{n}^{*}=\tau_{n}+$ $A\left(t_{n+1}\right)-A\left(t_{n}\right)$; the duality with the reflected random walk remains valid.

The duality between $\left\{X_{n}\right\}$ and $\left\{W_{n}\right\}$ extends to the case when $\left\{\left(B_{n}, \tau_{n}\right)\right\}$ forms a stationary sequence, but then its time reversal must be used in recursion (6) (see [1]).

Remark 3.2: Using a continuous-time analogue of Eq. [1] developed by Sigman [14] (or, because workload is a Markov process, by using Siegmund [13] duality), it can be shown that the duality in Eq. (4) holds for all $t \geq 0$ :

$$
P\left(X_{x}(t) \leq y\right)=P\left(V_{y}(t) \geq x\right), \quad t \geq 0,
$$


where $V_{y}(t)$ denotes the workload at time $t$ in the $\mathrm{M} / \mathrm{G} / 1$ queue with negative customers in which $V(0)=y \geq 0$. This means that the continuous-time $\{X(t)\}$ and $\{V(t)\}$ are duals of one another. This can be generalized further (using time reversal) to a time-stationary setting in which $\left\{\left(B_{n}, \tau_{n}\right)\right\}$ is defined from a timestationary marked point process or $\{A(t)\}$ from Remark 3.1 is a process with nonnegative stationary increments.

\section{Acknowledgment}

The first two authors gratefully acknowledge an insightful discussion with J. W. Cohen.

\section{References}

1. Asmussen, S. \& Sigman, K. (1996). Monotone stochastic recursions and their duals. Probability in the Engineering and Information Sciences 10: 1-20.

2. Boucherie, R.J. \& Boxma, O.J. (1996). The workload in the M/G/1 queue with work removal. Probability in the Engineering and Informational Sciences 10: 261-277.

3. Cohen, J.W. (1982). The single server queue. Amsterdam: North-Holland.

4. Cramér, H. (1955). Collective risk theory. Reprinted from the Jubilee Volume of Skandia Insurance Company, Esselte, Stockholm.

5. Fakinos, D. (1981). The G/G/1 queueing system with a particular queue discipline. Journal of the Royal Statistical Society B 43: 190-196.

6. Gelenbe, E. (1991). Product-form queueing networks with negative and positive customers. Journal of Applied Probability 28: 656-663.

7. Gelenbe, E. (1993). G-networks with triggered customer movement. Journal of Applied Probability 30: 742-748.

8. Jain, G. \& Sigman, K. (1996). Generalizing the Pollaczek-Khintchine formula to account for arbitrary work removal. Probability in the Engineering and Informational Sciences 10: 519-531

9. Jain, G. \& Sigman, K. (1996). A Pollaczek-Khintchine formulation for $M / G / 1$ queues with disasters. Journal of Applied Probability 33: 1191-1200.

10. Lukacs, E. (1970). Characteristic functions. London: Charles Griffin.

11. Niu, S.-C. (1988). Representing workloads in GI/G/1 queues through the preemptive-resume LIFO queue discipline. Queueing Systems 3: 157-178.

12. Prabhu, N.U. (1961). On the ruin problem of collective risk theory. Annals of Mathematical Statistics 32: 757-764.

13. Siegmund, D. (1976). The equivalence of absorbing and reflecting barrier problems for stochastically monotone Markov processes. Annals of Probability 4: 914-924.

14. Sigman, K. (1996). Continuous time stochastic recursions and duality. Manuscript.

15. Wolff, R.W. (1989). Stochastic modeling and the theory of queues. Englewood Cliffs, New Jersey: Prentice-Hall. 